\title{
Hiperparatiroidismo primario asociado a pancreatitis autoinmune, colangitis esclerosante y posible hipoglucemia autoinmune
}

\author{
Primary hyperparathyroidism associated with \\ autoimmune pancreatitis, sclerosing cholangitis \\ and probable autoimmune hypoglycemia
}

\author{
Sebastián Herrera, Juan Carlos Bravo, luis Guillermo Arango, \\ Carlos Alberto Cañas - Cali (Colombia)
}

\begin{abstract}
Resumen
Se informa el caso de una mujer de 42 años quien desarrolla pancreatitis autoinmune, colangitis esclerosante y probable hipoglucemia autoinmune, asociada con hiperparatiroidismo primario. (Acta Med Colomb 2012; 37: 80-82)

Palabras clave: pancreatitis autoinmune, colangitis esclerosante, hipoglucemia autoinmune, hiperparatiroidismo primario.
\end{abstract}

\begin{abstract}
We report the case of a 42 year old woman who developed autoimmune pancreatitis, sclerosing cholangitis and probable autoimmune hypoglycemia associated with primary hyperparathyroidism. (Acta Med Colomb 2012; 37: 80-82)

Keywords: autoimmune pancreatitis, sclerosing cholangitis, autoimmune hypoglycemia, primary hyperparathyroidism.
\end{abstract}

Dr. Sebastián Herrera: Residente de Medi-
cina Interna. Universidad CES-Fundación
Valle del Lili; Dr. Juan Carlos Bravo:
Patólogo; Fundación Valle del Lili; Dr.
Luis Guillermo Arango: Internista Endocri-
nólogo. Fundación Valle del Lili; Dr. Carlos
Alberto Cañas: Internista Reumatólogo,
Fundación Valle del Lili. Cali, Colombia.
Correspondencia. Dr. Carlos A. Cañas.
Fundación Valle del Lili. Cali, Colombia
E mail: cacd12@ @otmail.com
Recibido:01/XII/2010 Aceptado: 16/V/2012

\section{Introducción}

La asociación de pancreatitis autoimmune y colangitis esclerosante ha sido informada en la literatura médica, como una forma de conglomerado autoinmune de base fisiopatológica común (1). Una tercera condición asociada como es una hipoglucemia de posible base autoinmune no ha sido informada antes. Nosotros presentamos en este informe una paciente con esta asociación, además con el desarrollo de hipercalcemia severa asociada a un hiperparatiroidismo primario el cual se debió a la presencia de dos adenomas paratiroideos. El hiperparatiroidismo podría ser en algunos casos base fisiopatológica de activación de inmunidad humoral, y estar asociada con gamapatías y fenómenos de autoinmunidad $(2,3)$.

\section{Caso clínico}

Paciente mujer de 42 años, casada, dos hijos, con antecedentes de depresión mayor por lo cual había requerido hospitalización en tres ocasiones previas. Remitida de otra institución por episodios de hipoglucemia severa sintomá- tica, con glucometrías de $25 \mathrm{mg} / \mathrm{dL}$, a repetición y de difícil manejo pese a la infusión de dosis altas de dextrosa.

Revisión por sistemas: niega síntomas cutáneos, oculares, respiratorios, gastrointestinales, osteoarticulares y/o neurológicos previos.

Antecedentes personales: depresión mayor, que había requerido tres hospitalizaciones en el pasado. Recibía fluoxetina en forma irregular.

Examen físico de ingreso: PA 96/52 mmHg, FC 66', FR $22^{\prime}, \mathrm{T}^{\circ} 36^{\circ} \mathrm{C}$. Mal estado general, como dato positivo tenía dolor a la palpación difusa del abdomen. Los paraclínicos obtenidos al ingreso se presentan en la Tabla 1. Marcadores tumorales Ca19-9 y antígeno carcinoembrionario fueron negativos. Anticuerpos antinucleares, ENAs, anticuerpos anticardiolipina, factor reumatoide, anticuerpos antimitocondriales, anticuerpos antianhidrasa carbónica y antilactoferrina fueron informados negativos. IgG: $17.26 \mathrm{mg} / \mathrm{dL}$ (7-16mg/ dL). Complemento $\mathrm{C} 3$ disminuido. La IgG4, los anticuerpos antilactoferrina, antianhidrasacarbónica, antirreceptor de insulina y antiinsulina, no fue posible su realización. Los 
Tabla 1. Paraclínicos al ingreso y al momento del alta de la paciente. Se observa la corrección del hiperparatiroidismo y la hipoglucemia.

\begin{tabular}{|c|c|c|}
\hline Laboratorio & $\begin{array}{l}\text { Resultado } \\
\text { al ingreso }\end{array}$ & $\begin{array}{c}\text { Luego de } \\
\text { inmunosupresión } \\
\text { /paratiroidectomía }\end{array}$ \\
\hline Glucemia & $20 \mathrm{mg} / \mathrm{dL}$ & $91 \mathrm{mg} / \mathrm{dL}$ \\
\hline Calcio corregido & $12.7 \mathrm{mg} / \mathrm{dL}$ & $9.8 \mathrm{mg} / \mathrm{dL}$ \\
\hline Fósforo & $2.6 \mathrm{mg} / \mathrm{dL}$ & $4.3 \mathrm{mg} / \mathrm{dL}$ \\
\hline PTH & $227 \mathrm{ng} / \mathrm{L}$ & $66 \mathrm{ng} / \mathrm{L}$ \\
\hline Calcio en orina & $41 \mathrm{mg} / 24 \mathrm{~h}$ & \\
\hline Hemoglobina & $9.5 \mathrm{mg} / \mathrm{dL}$ & $12.3 \mathrm{mg} / \mathrm{dL}$ \\
\hline Hematocrito & $29.1 \%$ & $32 \%$ \\
\hline Leucocitos & $17.800 / \mathrm{mm}^{3}$ & $9.990 / \mathrm{mm}^{3}$ \\
\hline Neutrófilos & $73 \%$ & $78 \%$ \\
\hline Linfocitos & $16 \%$ & $20 \%$ \\
\hline Plaquetas & $492.000 / \mathrm{mm}^{3}$ & $312.000 / \mathrm{mm}^{3}$ \\
\hline Sodio & $138 \mathrm{mEq} / \mathrm{L}$ & \\
\hline Potasio & $3.71 \mathrm{mEq} / \mathrm{L}$ & \\
\hline Cloro & $109 \mathrm{mEq} / \mathrm{L}$ & \\
\hline Creatinina & $0.8 \mathrm{mg} / \mathrm{dL}$ & \\
\hline BUN & $2.0 \mathrm{mg} / \mathrm{dL}$ & \\
\hline Bilirrubina total & $0.4 \mathrm{mg} / \mathrm{dL}$ & \\
\hline Bilirrubina directa & $0.1 \mathrm{mg} / \mathrm{dL}$ & \\
\hline AST & $21.5 \mathrm{UI} / \mathrm{L}$ & \\
\hline ALT & $17.8 \mathrm{UI} / \mathrm{L}$ & \\
\hline Fosfatasa alcalina & $163.5 \mathrm{mg} / \mathrm{dL}$ & \\
\hline Albúmina & $2.1 \mathrm{~g} / \mathrm{dL}$ & $3.2 \mathrm{~g} / \mathrm{dL}$ \\
\hline TSH & $2.78 \mu \mathrm{U} / \mathrm{ml}$ & \\
\hline Amilasa & $132 \mathrm{U} / \mathrm{L}$ & \\
\hline Cortisol 8 a.m. & $22.3 \mu \mathrm{g} / \mathrm{dL}$ & \\
\hline Pro-péptido C & $\begin{array}{c}2.15 \mathrm{ng} / \mathrm{ml} \\
\text { (Rango: } 1.1-4.4 \mathrm{ng} / \mathrm{ml} \text { ) }\end{array}$ & \\
\hline Insulina & $\begin{array}{c}4.71 \mu \mathrm{U} / \mathrm{ml} \\
\text { (Rango: } 6-21 \mu \mathrm{U} / \mathrm{ml} \text { ) }\end{array}$ & \\
\hline IGF-1 (insulin like growth factor) & $\begin{array}{c}48.8 \mathrm{ng} / \mathrm{ml} \\
\text { (Rango: } 109-483 \mathrm{ng} / \mathrm{ml} \text { ) }\end{array}$ & \\
\hline Prolactina & $\begin{array}{c}111 \mathrm{ng} / \mathrm{ml} \\
\text { (Rango: } 2.0-25 \mathrm{ng} / \mathrm{ml} \text { ) }\end{array}$ & \\
\hline Glucagón & $\begin{array}{c}83 \mathrm{pg} / \mathrm{ml} \\
\text { (Rango: } 46-166 \mathrm{pg} / \mathrm{ml} \text { ) }\end{array}$ & \\
\hline
\end{tabular}

anticuerpos antimúsculo liso (ASMA) fueron informados como positivos

La escanografía abdominal demostró una masa pancreática hipointensa en T1, ligeramente hiperintensa en T2, de contornos pobremente definidos que infiltra la región de la unión entre la cabeza y el cuerpo, extendiéndose hacia la región periportal y el hilio hepático en forma difusa con signos de compromiso de la grasa periportal inclusive hasta la región portal izquierda, produciendo cambios irregulares y mal definidos en la intensidad de señal del parénquima hepático del segmento II y III. Obstrucción de la porta hepatis con circulación colateral. Los cambios infiltrativos peripan-

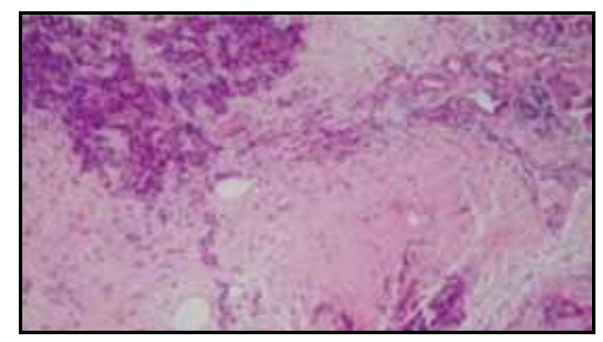

Figura 1. Tejido pancreático atrófico donde se observa fibrosis periductal, perilobular e intralobular con un infiltrado inflamatorio linfoplasmocitario. (H\&E a 10X).

creáticos rodeaban el tronco celiaco y la arteria hepática de manera circunferencial. Edema periportal y edema adyacente a la segunda y tercera porciones del duodeno. El páncreas distal a la lesión descrita presentaba cambios atróficos, con alteración difusa en su intensidad de señal.

Las biopsias hepática y pancreática bajo visión laparoscópica reportaron tejido hepático con extensos cambios fibróticos, infiltrado inflamatorio conformado por linfocitos, plasmocitos, eosinófilos y neutrófilos. Entre el tejido fibroconectivo se encontraba una proliferación de ductos, los cuales zonalmente tenían una fibrosis periductular con un proceso inflamatorio que se originaba en las células epiteliales ductulares con marcados cambios reparativos que mostraban atipia nuclear. En las regiones marginales depósitos de fibrina con severo infiltrado neutrofílico que formaba un absceso de detritus celulares y material biliar. En el tejido hepático conservado se apreciaba un infiltrado linfoplasmocitario en los espacios porta. Todo lo anterior se interpretó como una colangitis esclerosante. Los hallazgos en el páncreas fueron: pancreatitis esclerosante linfoplasmocítica con proliferación de fibroblastos que formaban haces densos y entre este tejido se encontraban escasos acinos pancreáticos con cambios celulares reactivos y presencia de un infiltrado inflamatorio conformado por linfocitos, plasmocitos y polimorfonucleares neutrófilos. Se interpreta como pancreatitis autoinmune (Figura 1).

La paciente tuvo una evolución tórpida, hipoglucemias a repetición, dolor abdominal y vómito. Con alto aporte de glucosa IV, antieméticos y nutrición enteral.

Se inician pulsos de metilprednisolona a dosis de $1 \mathrm{~g} /$ día por tres dosis y posterior cambio oral a razón de $2 \mathrm{mg} / \mathrm{kg} /$ día de prednisolona. La paciente mejora de las hipoglucemias lo cual está a favor de una etiología autoinmune. Se inicia inmunosupresión con ciclofosfamida a dosis de $750 \mathrm{mg} / \mathrm{m}^{2} \mathrm{IV}$.

Ante el aumento progresivo del calcio sérico se decide administrar dosis única de ácido zolendrónico. La gamagrafía de paratiroides reportó captación anormal en paratiroides inferiores derecha e izquierda (adenomas múltiples), por lo cual se lleva a paratiroidectomía subtotal. La patología reporta proliferación de células monomórficas de núcleos redondeados con citoplasma claro y eosinofílico que se agrupan en nidos contenidos entre pequeños canales, con formación de túbulos y cordones, libres de tejido adiposo, 
con pseudocápsula y tejido paratiroideo comprimido. Tejido glándula paratiroides inferior derecha e inferior izquierda consistente con adenoma.

Con las estrategias terapéuticas realizadas la paciente mejora tanto en la clínica como en los parámetros paraclínicos (Tabla 1), por lo cual se da de alta con miras a continuar manejo ambulatorio. La paciente no vuelve a controles por problemas de tipo administrativo, y luego se obtiene información de su deceso cuya causa probable fue el retiro de la medicación por falta de suministro.

\section{Discusión}

La pancreatitis autoinmune es una inflamación crónica del páncreas con características clínicas, radiológicas e histológicas particulares pero con una patogénesis poco comprendida. Puede asociarse con manifestaciones extrapancreáticas como colangitis esclerosante, fibrosis retroperitoneal y sialoadenitis esclerosante (4). Se acompaña de insuficiencia exocrina pancreática en $88 \%$ de los casos y disfunción endocrina en $67 \%$. Esta es una entidad rara aunque con un número de reportes en incremento en los últimos 10 años, su prevalencia en los casos de pancreatitis crónica puede ir del 5 a $11 \%$ según la serie (5). En nuestro caso se cumplieron los criterios diagnósticos para esta condición (6), incluyendo aspectos imagenológicos, histológicos y de respuesta inmune. Se presentaron dos condiciones autoinmunes asociadas, colangitis esclerosante, cuyo diagnóstico fue basado en los hallazgos histológicos, y una hipoglucemia que respondió al uso de esteroides, muy posiblemente de tipo autoinmune. Desafortunadamente no contamos en nuestro medio con la técnica para la realización de anticuerpos antiinsulina y su receptor, fundamentales para confirmar este diagnóstico (7).

Llama la atención la asociación adicional con hiperparatiroidismo primario por la presencia de adenomas de las paratiroides inferiores (adenoma múltiple), situación que sucede sólo en 2-12\% de los casos de hiperparatiroidismo primario (8). Un caso similar al de nuestra paciente con pancreatitis autoinmune, colangitis esclerosante, hipoglucemia autoinmune e hiperpatiroidismo fue informado por Pallais $\mathrm{J}$, et al. (9), pero en este caso se demostró una forma de hiperpatiroidismo no relacionado con neoplasia sino con una condición de hiperparatiroiditis con anticuerpos estimulantes de receptores sensores de calcio.

Una posible explicación de la condición autoinmune asociada a hiperactividad paratiroidea podría ser el estímulo de la hormona paratiroidea para la producción de interleuquina-6 (IL-6) por parte de células estromales, como el osteoblasto (10). La IL-6 tiene actividades pro y antiinflamatorias, además, es primordial para la proliferación y diferenciación de células B, responsables de la respuesta inmune de tipo humoral y la generación consecuente de anticuerpos (11).

Con el presente informe se desea demostrar una forma compleja de autoinmunidad (pancreatitis autoinmune, colangitis esclerosante, hipoglucemia posiblemente autoinmune), asociada a hiperparatiroidismo primario. Se postula una posible explicación fisiopatológica a través de la acción de la hormona paratiroidea sobre células estromales tipo osteoblasto, sobre producción de IL-6 y activación de linfocitos B.

\section{Referencias}

1. Nakazawa T, Ohara H, Sano H, Ando T, Aoki S, Kobayashi S, et al. Clinical differences between primary sclerosing cholangitis and sclerosing cholangitis with autoimmune pancreatitis. Pancreas 2005; 30: 20-5.

2. Cañas CA. Relación entre hiperparatiroidismo y gamapatía monoclonal. Acta Med Colomb 2007; 32: 219-22.

3. Cañas CA, Echeverri AF, Arango LG, Anaya JM. Total recovery of monoclonal gammopathy after parathyroidectomy in a patient with Sjögren's syndrome and hyperparathyroidism (En prensa).

4. Kamisawa T, Egawa N, Nakajima H, Tsuruta K, Okamoto A. Extrapancreatic lesions in autoimmune pancreatitis. J Clin Gastroenterol 2005; 39: 904-907.

5. Kim KP, Kim M, lee YJ. Clinical characteristics of 17 cases of autoimmune chronic pancreatitis. Korean. J Gastroenterol 2004; 43: 112-119.

6. Kim K-P, Kim M-H, Kim JC, lee SS, Seo DW, Lee SK. Diagnostic criteria for autoimmune chronic pancreatitis revisited. World J Gastroenterol 2006; 12: 2487-2496.

7. Taylor SI, Barbetti F, Accili D, Roth J, Gorden P. Syndromes of autoimmunity and hypoglycemia: autoantibodies directed against insulin and its receptor. Endocrinol Metab Clin North Am 1989; 18:123-143.

8. Fraser W. Hyperparathyroidism. Lancet 2009; 374: 145-58.

9. Pallais J, Kifor O, Chen Y, Slovik D, Brown E. Acquired hypocalciuric hypercalcemia due to autoantibodies against the calcium-sensing receptor. $N$ Engl $J$ Med 2004; 351: 362-9.

10. Khan A, Bilezikian J. Primary hyperparathyroidism: pathophysiology and impact on bone. CMAJ 2000; 163: 184-7.

11. Jelinek DF, Lipsky PE. Regulation of human B lymphocyte activation, proliferation, and differentiation. Adv Immunol 1987; 40: 1-59. 